\title{
Rotary-Wing UAVs Trajectory Planning by Distributed Linear MPC with Reconfigurable Communication Network Topologies
}

\author{
Alexandra Grancharova*,**, Esten I. Grotli***, Tor A. Johansen***** \\ * Institute of System Engineering and Robotics, Bulgarian Academy of Sciences, Acad. G. Bonchev str., Bl.2, P.O.Box 79, \\ Sofia 1113, Bulgaria (Tel: +359889625010; e-mail: alexandra.grancharova@abv.bg) \\ ** Department of Industrial Automation, University of Chemical Technology and Metallurgy, \\ 8 Sv. Kliment Ohridski Blvd., Sofia 1756, Bulgaria \\ *** Department of Engineering Cybernetics, Norwegian University of Science and Technology, 7491 Trondheim, Norway \\ (e-mail: Esten.Ingar.Grotli@itk.ntnu.no) \\ **** Center for Autonomous Marine Operations and Systems (AMOS), Department of Engineering Cybernetics, \\ Norwegian University of Science and Technology, 7491 Trondheim, Norway (e-mail: Tor.Arne.Johansen@itk.ntnu.no)
}

\begin{abstract}
In this paper, a distributed approach to Model Predictive Control (MPC)-based trajectory planning for rotary-wing UAV (Unmanned Aerial Vehicle) communication network topologies under radio path loss constraints is proposed. The goal is to find trajectories that are safe with respect to grounding and collision, fuel efficient and satisfy criteria for communication such that the UAVs form chains to multiple targets with given radio communication capacities. The MPC-based optimization subproblems are computed autonomously within each UAV, using convex quadratic programming, with the requirement that each UAV communicates its current measured position to all other UAVs. In addition, a simple coordination between UAVs allows for the communication network topology to be reconfigured in case of failures or radio path loss changes. The control performance of the distributed linear MPC trajectory planning is studied based on a simulation case with four UAVs and two targets.
\end{abstract}

Keywords: Autonomous vehicles, Radio-communication, Path planning, Predictive control, Distributed control, Constraints, Multi-vehicle coordination.

\section{INTRODUCTION}

This research is motivated by emerging applications of UAVs (Unmanned Aerial Vehicles) such as Arctic offshore ice monitoring and other complex long range multivehicle missions in areas without a permanent communication infrastructure. The problem of path planning for UAVs has been considered in several papers. For instance, in Burdakov et al. (2009), relay chains are generated solving the all hops optimal path graph search problem. In Grøtli and Johansen (2012), a Mixed-Integer Linear Programming approach for path and mission planning under radio communication constraints for UAVs is developed. Location and movement of UAVs are optimized in Han et al. (2009), in order to improve the connectivity of a wireless network. In Dixon and Frew (2012), a decentralized mobility control algorithm for an optimal end-to-end communication chain using a team of unmanned aircrafts acting solely as communication relays is presented. In Le Ny et al. (2012), an optimization-based approach to the deployment of an ad-hoc wireless network between UAVs is proposed, which decomposes into two components, one for position optimization and one for communication optimization, coupled via a set of Lagrange multipliers. In Moses Sathyaraj et al. (2008), path planning and path finding algorithms for multiple UAVs are studied. In Michael and Kumar (2011), a framework based on a lowdimensional abstraction is developed that enable a group of aerial robots to maintain a formation while avoiding collisions. In Gan and Sukkarieh (2011), a novel approach for
multi-UAV target search using a decentralized gradient-based optimization is proposed, where the gradient is explicitly formulated.

Model Predictive Control (MPC) is an optimization-based method for control that can handle state and input constraints (Mayne et al. (2000)). In the case of full control, there is no path planned in advance and the objective usually consists in minimizing fuel consumption, while meeting the overall mission objectives. In Dunbar and Murray (2006), a distributed MPC approach to multi-vehicle formation stabilization is proposed in which each subsystem is assigned its own optimal control problem, optimizes only for its own control at each update, and exchanges information only with neighboring subsystems. In Kuwata and How (2011), a decentralized MPC approach for path planning for cooperative UAVs in the presence of bounded disturbances is developed. In Grancharova et al. (2012a), a distributed nonlinear MPC-based trajectory planning approach for a fixed-wing UAVs communication chain is proposed (a single target is assumed), which explicitly incorporates constraints on radio communication path losses. In (Grancharova et al. (2012b), the distributed MPC-based trajectory planning problem for a group of UAVs is solved by applying the dynamic dual decomposition method (Giselsson and Rantzer (2010)). In Shin and Kim (2009), centralized and decentralized MPC methods to solve a re-planning problem are compared. 
In this paper, a distributed linear MPC approach to solve the trajectory planning problem for rotary-wing UAVs is proposed, where the objective of the UAV system is to form a communication network to multiple targets with given radio communication capacities. The approach explicitly incorporates constraints on radio communication path losses, computed by using SPLAT! (Maglicane (2010)). For terrain collision avoidance, at each time sample the terrain below each UAV and the communication path losses are approximated with linear functions of the spatial coordinates. This leads to linear MPC sub-problems, which are solved by using convex quadratic programming. A simple coordination between UAVs allows for the communication topology to be reconfigured in case of failures or changes in radio path loss. We assume the reconfiguration is handled by a separate system, and consider only the trajectory planning. The performance of the distributed linear MPC trajectory planning is studied on a simulation case with four UAVs and two targets.

\section{RECONFIGURABLE COMMUNICATION CHAINS BY DISTRIBUTED LINEAR MPC}

\subsection{UAVs model in ENU coordinate system}

The East-North-Up (ENU) coordinate frame is a local geodetic coordinate system whose tangent plane is fitted to the geodetic reference ellipse at some convenient point for local measurements. The $x$ axis points towards east, the $y$ axis points towards true north and the $z$ axis completes the right handed orthogonal frame by pointing away from the Earth perpendicular to the reference ellipsoid.

It is assumed that the $i$-th UAV is described by the following discrete time model in the ENU coordinate system:

$$
p_{i}(t+1)=p_{i}(t)+T_{\mathrm{S}} v_{i}(t),
$$

$i=1, \ldots, M$, where $M$ is the number of UAVs, $p_{i}(t)=\left[x_{i}(t), y_{i}(t), z_{i}(t)\right] \quad$ and $\quad v_{i}(t)=\left[v_{i x}(t), v_{i y}(t), v_{i z}(t)\right]$, with $x_{i}, y_{i}, z_{i}$ and $v_{i x}, v_{i y}, v_{i z}$ being the positions and the velocities along the orthogonal axes in the local ENU coordinate reference frame, and $T_{\mathrm{S}}$ is the sampling period.

We consider the case where $M$-number of rotary-wing UAVs are tasked to form $L$-number of communication chains between one base station and $L$-number of moving targets. The path-planning problem for the UAVs is formulated as an MPC problem, where the optimization is performed over velocity sequences $V_{i}=\left[v_{i, t}, v_{i, t+1}, \ldots, v_{i, t+N-1}\right], i=1, \ldots, M$ along a prediction horizon with length $N$. The predicted position $p_{i, t+k \mid t}$ of the $i$-th $\mathrm{UAV}$, given the initial position $p_{i, t \mid t}=p_{i}(t)$ and the velocity sequence $V_{i}$ is:

$$
p_{i, t+k+1 \mid t}=p_{i, t+k \mid t}+T_{\mathrm{S}} v_{i, t+k}, k=0,1, \ldots, N-1
$$

\subsection{Constraints}

The following constraints are imposed on the UAVs.

1. Constraints on the velocity vectors

Assume that the maximum allowed speed for the $i$-th rotarywing UAV is $\bar{S}_{i}, i=1, \ldots, M$. This upper bound is respected by imposing the following hard constraints on the elements $v_{i x}, v_{i y}, v_{i z}$ of the velocity vector $v_{i}$ :

$$
\begin{aligned}
& -\bar{v}_{i} \leq v_{i x, t+k} \leq \bar{v}_{i},-\bar{v}_{i} \leq v_{i y, t+k} \leq \bar{v}_{i},-\bar{v}_{i} \leq v_{i z, t+k} \leq \bar{v}_{i} \\
& k=0,1, \ldots, N-1
\end{aligned}
$$

where $\bar{v}_{i}=\sqrt{\bar{S}_{i}^{2} / 3}$

\section{Acceleration constraints}

The acceleration constraints for the $i$-th UAV are:

$$
\begin{aligned}
& -\Delta_{i} \mathbf{1} \leq\left(v_{i, t}-v_{i}(t-1)\right) / T_{\mathrm{S}} \leq \Delta_{i} \mathbf{1} \\
& -\Delta_{i} \mathbf{1} \leq\left(v_{i, t+k+1}-v_{i, t+k}\right) / T_{\mathrm{S}} \leq \Delta_{i} \mathbf{1}, k=0,1, \ldots, N-2
\end{aligned}
$$

where $\mathbf{1}=[1,1,1]^{T}$ and $\Delta_{i}, i=1, \ldots, M$ are known constants.

\section{Anti-grounding and air space constraints}

The following constraints are imposed on the position of the $i$-th UAV in the ENU coordinate system:

$$
\begin{aligned}
& \underline{x}_{i}-q_{x, i} \leq x_{i, t+k \mid t} \leq \bar{x}_{i}+q_{x, i}, \underline{y}_{i}-q_{y, i} \leq y_{i, t+k \mid t} \leq \bar{y}_{i}+q_{y, i} \\
& \hat{h}_{i}^{\text {terr }}\left(p_{i, t+k \mid t}\right)+\Delta h-q_{z, i} \leq z_{i, t+k \mid t} \leq \bar{z}_{i}+q_{z, i} \\
& k=1, \ldots, N
\end{aligned}
$$

where $x_{i, t+k \mid t}, y_{i, t+k \mid t}, z_{i, t+k \mid t}$ are the predicted coordinates of the $i$-th UAV by using the model (2) for a given velocity sequence $V_{i}, \underline{x}_{i}, y_{i}$ and $\bar{x}_{i}, \bar{y}_{i}, \bar{z}_{i}$ are lower and upper bounds,

$\hat{h}_{i}^{\text {terr }}\left(p_{i, t+k \mid t}\right)$ represents the approximate height of the terrain below the $i$-th UAV and within its maximal reach distance for the specified time horizon, and $\Delta h$ is a safety distance from ground. Here, $q_{x, i}, q_{y, i}, q_{z, i} \geq 0$ are slack variables, which are introduced in order to avoid infeasibility. The constraints imposed on the coordinate $z$ are such that the terrain collision avoidance requirement is met.

The height of the terrain below the $i$-th UAV is upper approximated with the following linear function:

$$
\hat{h}_{i}^{\text {terr }}\left(p_{i, t+k \mid t}\right)=a_{i}^{T}\left(x_{i, t \mid t}, y_{i, t \mid t}\right)\left[\begin{array}{lll}
x_{i, t+k \mid t} & y_{i, t+k \mid t} & 1
\end{array}\right]^{T}
$$

Here, the vector $a_{i}\left(x_{i, t \mid t}, y_{i, t \mid t}\right) \in \mathbb{R}^{3}$ contains the coefficients, which depend on the initial position of the $i$-th UAV and they are obtained by solving a Linear Programming (LP) problem (see Grancharova et al. (2012a)).

\section{Constraints on radio communication path losses}

The task where $M$-number of rotary-wing UAVs are assigned to form $L$-number of primary communication chains between one base station and $L$-number of targets is considered. In each chain, it is assumed that only one UAV communicates with the base station and only one UAV should communicate with the respective target. All neighbouring UAVs within the chain should be able to communicate with each other (with higher priority) and with the neighbouring UAVs from the other chains (with lower priority). The last requirement is necessary in order for the chains to be quickly reconfigured in case some of the UAVs loose connectivity. Let also the base station be indexed as the node 0 and the $L$-number of targets be indexed as the $M+1, M+2, \ldots, M+L$ nodes. The connectivity constraints represent constraints on the radio path losses in the communication between the UAVs. The radio path losses are computed by using SPLAT! (Maglicane 
(2010), McMellen (2010)) and are approximated by linear functions at each time sample. SPLAT! uses elevation data to calculate field strength and path loss based on the LongleyRice Irregular Terrain Model (Longley and Rice (1968)).

It is assumed that the communication topology is specified by the matrix $\Psi=\left\{\psi_{i, j}\right\}, i, j=1, \ldots, M \quad$ with $\quad \psi_{i, i}=0$, $i=1, \ldots, M$. The elements $\psi_{i, j}, i, j=1,2, \ldots, M, i \neq j$ can have the following values:

- $\psi_{i, j}=0$, if the $i$-th UAV and the $j$-th UAV are not neighbours;

- $\quad \psi_{i, j}=1$, if the $i$-th UAV and the $j$-th UAV are neighbours from the same chain;

- $\psi_{i, j}=0.5$, if the $i$-th UAV and the $j$-th UAV are neighbours from different communication chains.

In the formulation of the MPC sub-problem for the $i$-th UAV, only constraints on the communication path losses to the neighbouring UAVs will be imposed, which correspond to $\psi_{i, j} \neq 0, j=1,2, \ldots, M$. Also, the weighting coefficient for the slack variable in a given path loss constraint should be much larger if $\psi_{i, j}=1$ than the case when $\psi_{i, j}=0.5$. This is to distinguish the different priorities in the communication between UAVs. The priority is higher when it involves UAVs from the same chain than the case when UAVs are from neighbouring chains.

In addition to the topology matrix, the following vectors are specified:

- $\varphi=\left[\varphi_{1}, \varphi_{2}, \ldots, \varphi_{M}\right]$ with $\varphi_{i}=1$ if the $i$-th UAV is assigned to communicate with the base station and $\varphi_{i}=0$, otherwise.

- $\xi=\left[\xi_{1}, \xi_{2}, \ldots, \xi_{M}\right]$, where $\xi_{i}=0$ if the $i$-th UAV is not assigned to communicate with any of the targets, otherwise, $\xi_{i} \in\{1,2, \ldots, L\}$ is the index of the target the $i$ th UAV should communicate with.

Thus, for the $i$-th UAV, the following constraints on the radio communication path losses are imposed:

- Constraint on radio communication path loss to the base station, if $\varphi_{i} \neq 0$ :

$$
\hat{c}_{i-0, t+k \mid t}\left(p_{i, t+k \mid t}\right) \leq \bar{c}_{i-0}+q_{i-0}, k=1, \ldots, N
$$

where $\hat{c}_{i-0, t+k \mid t}\left(p_{i, t+k \mid t}\right)$ represents the approximate path loss in the communication from the base station to the $i$-th UAV and $\bar{c}_{i-0}$ is the maximum allowed path loss. Here, $q_{i-0} \geq 0$ is a slack variable, which is introduced in order to avoid infeasibility. The path loss is upper approximated with the following linear function:

$$
\hat{c}_{i-0, t+k \mid t}\left(p_{i, t+k \mid t}\right)=b_{i-0}^{T}\left(p_{i, t \mid t}\right)\left[p_{i, t+k \mid t}^{T} 1\right]^{T}
$$

where the vector $b_{i-0}\left(p_{i, t \mid t}\right) \in \mathbb{R}^{4}$ contains the coefficients $b_{i-0, j}, j=1, \ldots, 4$, which depend on the initial position of the $i$-th UAV and the fixed base station position. They are obtained by solving the following LP problem:

$$
\begin{aligned}
& \min _{b_{i-0, j}, j=1, \ldots, 4}\left(b_{i-0,1} x_{i}^{\text {center }}+b_{i-0,2} y_{i}^{\text {center }}+b_{i-0,3} z_{i}^{\text {center }}+b_{i-0,4}\right)(9) \\
& \text { subject to } B_{i-0}\left[\begin{array}{llll}
b_{i-0,1} & b_{i-0,2} & b_{i-0,3} & b_{i-0,4}
\end{array}\right]^{T} \geq c_{i-0}
\end{aligned}
$$

Here, the matrix $B_{i-0}=\left[\begin{array}{lll}x_{i, j} & y_{i, j} & z_{i, j}\end{array}\right], j=1, \ldots, \tilde{N}_{i}$ contains the coordinates of a given number of points, which are within the maximal reach distance $d_{i, \max }$ for the $i$-th UAV, the vector $c_{i-0}=\left[c_{i-0, j}\right], j=1, \ldots, \tilde{N}_{i}$ contains the path losses between these points and the base station (computed with SPLAT!), and $x_{i}^{\text {center }}, y_{i}^{\text {center }}, z_{i}^{\text {center }}$ are the mean coordinates of these points. The maximal reach distance is determined as $d_{i, \max }=N \bar{S}_{i}$, where $N$ is the time horizon in the MPC problem and $\bar{S}_{i}$ is the maximal allowed speed of the $i$-th UAV.

- Constraints on radio communication path loss to the $\boldsymbol{j}$ th $\mathbf{U A V}$ if $\psi_{i, j} \neq 0, j=1, \ldots, M$ :

$\underline{c}_{i-j}-q_{i-j} \leq \hat{c}_{i-j, t+k \mid t}\left(p_{i, t+k \mid t}, p_{j, t \mid t}\right) \leq \bar{c}_{i-j}+q_{i-j}, k=1, \ldots, N$

where $\hat{c}_{i-j, t+k \mid t}\left(p_{i, t+k \mid t}, p_{j, t \mid t}\right)$ represents the approximate path loss in the communication from the $i$-th UAV to the $j$-th $\mathrm{UAV}, c_{i-j}$ and $\bar{c}_{i-j}$ are the minimal and the maximum allowed path losses, respectively, and $q_{i-j}$ is a slack variable. The lower bound $\underline{c}_{i-j}$ on the path loss is introduced in order to avoid collision between the UAVs. The rationale behind this is that the communication path loss between two UAVs will depend on the terrain and the distance between them. Therefore, it is possible to avoid collision by keeping the path loss above the specified minimal value. The path loss in (9) is upper approximated with the following linear function:

$$
\hat{c}_{i-j, t+k \mid t}\left(p_{i, t+k \mid t}, p_{j, t \mid t}\right)=b_{i-j}^{T}\left(p_{i, t \mid t}, p_{j, t \mid t}\right)\left[p_{i, t+k \mid t}^{T} 1\right]^{T}
$$

The coefficients $b_{i-j}\left(p_{i, t \mid t}, p_{j, t \mid t}\right) \in \mathbb{R}^{4}$ depend on the initial positions of the $i$-th UAV and the $j$-th UAV.

- Constraint on radio communication path loss to the $\boldsymbol{l}$ th target station, if $\xi_{i} \neq 0, l=\xi_{i}$ :

$\hat{c}_{i-(M+l), t+k \mid t}\left(p_{i, t+k \mid t}, p_{M+l, t \mid t}\right) \leq \bar{c}_{i-(M+l)}+q_{i-(M+l)}, k=1, \ldots, N(13)$ where $\hat{c}_{i-(M+l), t+k \mid t}\left(p_{i, t+k \mid t}, p_{M+l, t \mid t}\right)$ is the approximate path loss in the communication from the $i$-th UAV to the $l$-th target station (denoted as node $M+l), \bar{c}_{i-(M+l)}$ is the maximum allowed path loss, and $q_{i-(M+l)} \geq 0$ is a slack variable. The path loss is upper approximated with the following linear function:

$$
\begin{aligned}
\hat{c}_{i-(M+l), t+k \mid t}\left(p_{i, t+k \mid t}, p_{M+l, t \mid t}\right) & \\
= & b_{i-(M+l)}^{T}\left(p_{i, t \mid t}, p_{M+l, t \mid t}\right)\left[p_{i, t+k \mid t}^{T} 1\right]^{T}
\end{aligned}
$$

The coefficients $b_{i-(M+l)}\left(p_{i, t \mid t}, p_{M+l, t \mid t}\right) \in \mathbb{R}^{4}$ depend on the initial positions of the $i$-th UAV and the $l$-th target.

The coefficients in the approximating linear functions (12) and (14) are obtained by solving LP problems, which are similar to the LP problem (9)-(10). 


\subsection{Reconfigurable communication chains by distributed linear MPC}

With the distributed MPC approach, the approximated path losses in (7), (11), and (13) are computed as dependent on the predicted coordinates of the considered UAV only, i.e. it is assumed that the positions of the neighbouring UAVs do not change during predictions when solving the corresponding MPC sub-problem. It is assumed that each UAV communicates its current measured position to its neighbouring UAVs. Thus, for the current measured positions $p_{r}(t), r=1, \ldots, M$ of the UAVs, the MPC path planning solves a distributed optimization problem consisting of the following sub-problems:

Problem $P_{i}, i=1,2, \ldots, M$ :

(MPC sub-problem for the $i$-th UAV)

$$
J_{i}^{*}=\min _{V_{i}, q_{i}} J_{i}\left(V_{i}, q_{i}\right)
$$

subject to $p_{r, t \mid t}=p_{r}(t), r=1, \ldots, M$, constraints (2)-(5), and:

$$
\hat{c}_{i-0, t+k \mid t}\left(p_{i, t+k \mid t}\right) \leq \bar{c}_{i-0}+q_{i-0}, k=1, \ldots, N
$$

for $\varphi_{i} \neq 0$

$\underline{c}_{-j-j}-q_{i-j} \leq \hat{c}_{i-j, t+k \mid t}\left(p_{i, t+k \mid t}, p_{j, t \mid t}\right) \leq \bar{c}_{i-j}+q_{i-j}, k=1, \ldots, N$

for $\psi_{i, j} \neq 0, j=1, \ldots, M$

$\hat{c}_{i-(M+l), t+k \mid t}\left(p_{i, t+k \mid t}, p_{M+l, t \mid t}\right) \leq \bar{c}_{i-(M+l)}+q_{i-(M+l)}, k=1, \ldots, N$

for $\xi_{i} \neq 0, l=\xi_{i}$

$q_{i} \geq 0$

with the cost function:

$$
J_{i}\left(V_{i}, q_{i}\right)=\sum_{k=0}^{N-1}\left[\alpha_{i}\left\|v_{i, t+k}\right\|^{2}+q_{i}^{T} \Lambda_{i} q_{i}\right]
$$

Here, $q_{i}$ is the vector of all slack variables, $\alpha_{i}$ is a weighting coefficient and $\Lambda_{i}$ is a weighting matrix. The first term in (20) represents an approximation to the UAV energy consumption.

The linear MPC sub-problem, formulated above, can be solved by convex quadratic programming.

Initially, all UAVs are located near the base station and the communication chains to the multiple targets are preassigned. At the same time, a simple coordination procedure is performed by each UAV, which allows reconfiguration of the communication chains in case of failure (for example, a lost communication with some UAV). This situation can be recognized if a UAV in some communication chain did not communicate its current position to the other UAVs. Then, this UAV will be considered as disconnected and its task will be taken over by a neighbouring UAV in the same chain (this is implemented as Procedure 1, described below). However, if it becomes clear after some time that the reduced number of UAVs in this chain can not maintain the communication from the base station to the respective target, a UAV from a neighbouring chain will join that chain (implemented as Procedure 2, given below). In such way, this UAV will be used in both chains. The reconfiguration of the communication chains will reflect only on the values of the weighing coefficients for the slack variables in the constraints on the communication path losses in some of the MPC subproblems.

The following procedure is applied when the $i$-th UAV did not receive the current position of some other UAV in the same chain.

\section{Procedure 1:}

1. for $j=1,2, \ldots, M$ do

2. if $\psi_{i, j}=1$ then

3. The $i$-th should receive the current position of the $j$ th UAV. In case this is successful, then $\eta:=1$. Otherwise, $\eta:=0$.

4. end

5. if $\psi_{i, j}=1$ and $\eta=0$ then

6. The $i$-th UAV takes the task of the $j$-th UAV. The topology matrix $\Psi$ and the vectors $\varphi$ and $\xi$ are rearranged. Terminate.

\section{7. end}

8. end

The next procedure is performed in case the $i$-th UAV is tasked to communicate with both the base station and one target. This can be the result when there have been totally 2 UAVs in the chain and the communication with one of them has been lost. In this case, a UAV which is both neighbouring to the $i$-th UAV and communicates with the base station will be found. Such UAV, in addition to its own chain, will join the chain of the $i$-th UAV, where its task will be to communicate with the base station and the $i$-th UAV.

\section{Procedure 2:}

1. if some of the radio communication path losses, associated to the $i$-th UAV are close to the maximum values, then

2. for $j=1,2, \ldots, M$ do

3. if $\psi_{i, j}=0.5$ and $\varphi_{j}=1$ then

4. Assign the $j$-th UAV to join the communication chain of the $i$-th UAV, i.e. $\psi_{i, j}:=1$. Terminate.

5. end
$6 . \quad$ end
7. end

\section{SIMULATION EXAMPLE}

In this section we use four UAVs to form two radio communication chains from a base station to a moving target (T1) and to a stationary target (T2) (see Fig. 1). The base station and the target T2 are located at $(6000,1000,150)$ and $(5000,6000,10)$, respectively. The initial position of target $\mathrm{T} 1$ is $(100,6000,10)$ and its speed is $8[\mathrm{~m} / \mathrm{s}]$ towards east. The initial positions of the UAVs are:

$$
\begin{aligned}
& p_{1}=(5500,1500,300), p_{2}=(5750,1500,300) \\
& p_{3}=(5500,1750,300), p_{4}=(5750,1750,300)
\end{aligned}
$$

The parameter values for the UAVs, as well as for the optimization problem are given in Table 1. In this example, the base station is denoted as node 0 , the target $\mathrm{T} 1$ - as node 5 , and the target $\mathrm{T} 2$ - as node 6 . 
Table 1. Parameter values.

\begin{tabular}{|cc|}
\hline \multicolumn{2}{|c|}{ Parameter } \\
\hline$\underline{x_{i}}, \bar{x}_{i}, \underline{y}_{i}, \bar{y}_{i}, \bar{z}_{i}, i=1,2,3,4$ & $500,5800,1250,5500,600$ \\
\hline $\bar{S}_{1}, \bar{S}_{2}, \bar{S}_{3}, \bar{S}_{4}$ & $25[\mathrm{~m} / \mathrm{s}], 25[\mathrm{~m} / \mathrm{s}], 33[\mathrm{~m} / \mathrm{s}], 33[\mathrm{~m} / \mathrm{s}]$ \\
\hline$\Delta_{i}, i=1,2,3,4$ & $1\left[\mathrm{~m} / \mathrm{s}^{2}\right]$ \\
\hline$\Delta h$ & $100[\mathrm{~m}]$ \\
\hline $\bar{c}_{1-0}, \bar{c}_{2-0}, \bar{c}_{3-5}, \bar{c}_{4-6}$ & $108[\mathrm{~dB}], 108[\mathrm{~dB}], 112[\mathrm{~dB}], 108[\mathrm{~dB}]$ \\
\hline$\underline{c}_{i-j}, \bar{c}_{i-j}, i, j=1,2,3,4, i \neq j$ & $85[\mathrm{~dB}], 102[\mathrm{~dB}]$ \\
\hline$T_{\mathrm{S}}, N$ & $1[\mathrm{~s}], 10$ \\
\hline$\alpha_{i}, i=1,2,3,4$ & 0.1 \\
\hline
\end{tabular}

The distributed linear MPC approach, described in the previous section, is applied and the results are presented in Fig. 5 to Fig. 18.

In the time period $[0 ; 700]$ all UAVs are used (topology shown in Fig. 1 and Fig. 2). At time 700, UAV4 is out of use and the communication chains are reconfigured (that is why all graphics, associated to UAV4, are depicted only until time 700 (Figs. 6, 7, 12, 14, 15, 17)). Then, Procedure 1 is applied and in the period $[700 ; 780]$, the network topology from Fig. 3 is used, where UAV2 also takes the taks of UAV4. Thus, UAV2 should be able to communicate with both the base station and the target T2. Since with this scenario, the communication path loss between the UAV2 and the base station approaches the maximal allowed limit (see Fig. 9), at time 780 Procedure 2 is used and the communication topology is reconfigured again, as it is shown in Fig. 4. In this topology, UAV1 is used in both chains. It can be seen from Fig. 18 that UAV2 manages to communicate to the target T2. It can also be observed from Fig. 16 that UAV3 in the other chain establishes communication with the target $\mathrm{T} 1$. From Fig. 10 to Fig. 15 it can be noticed that the communication path losses between UAVs in a defined chain never exceed the maximum allowed values.

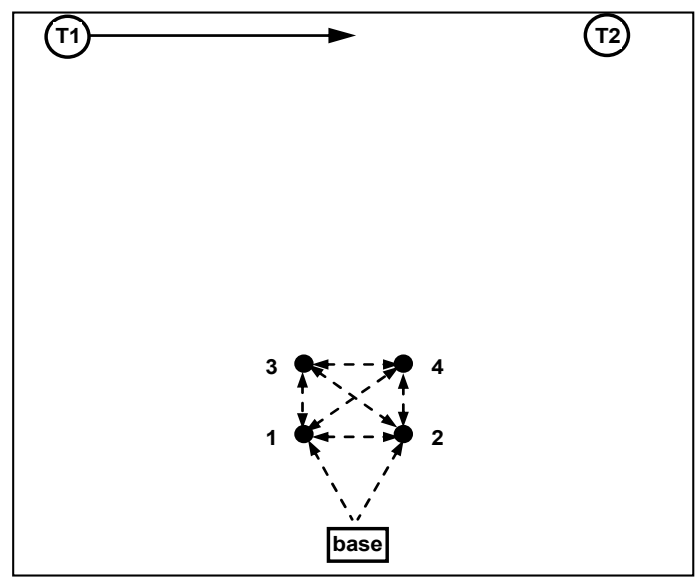

Fig. 1. Initial positions of the UAVs, the base, and the targets.

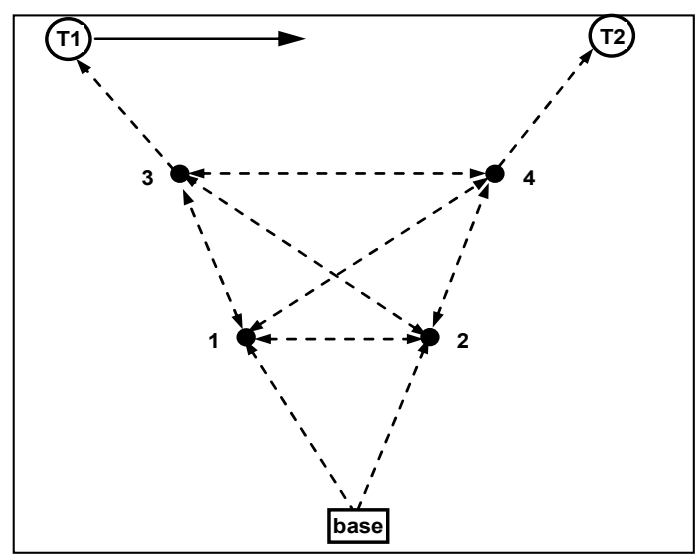

Fig. 2. Two communication chains: Base-UAV1-UAV3-T1, Base-UAV2-UAV4-T2.

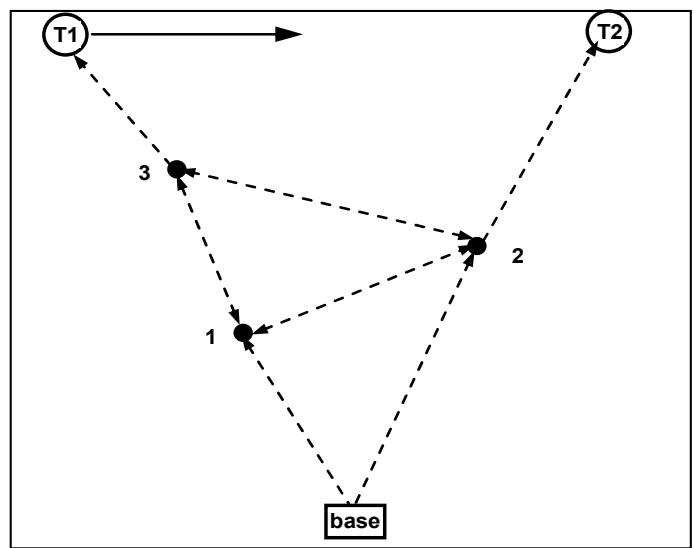

Fig. 3. Two communication chains: Base-UAV1-UAV3-T1, Base-UAV2-T2.

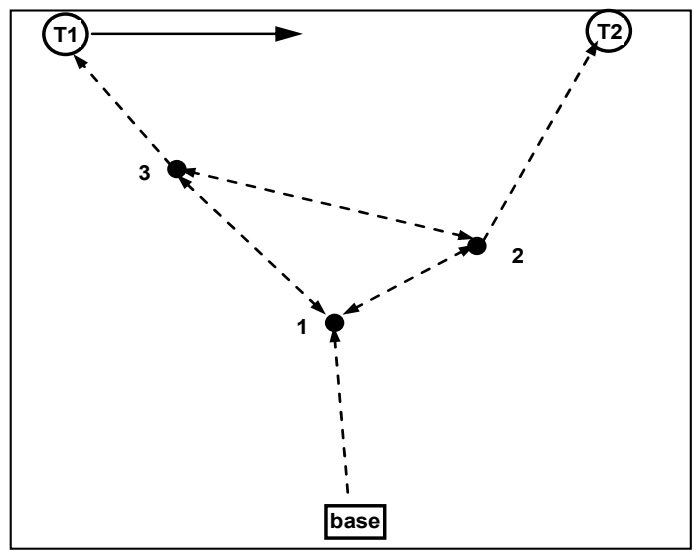

Fig. 4. Two communication chains: Base-UAV1-UAV3-T1, Base-UAV1-UAV2-T2. 


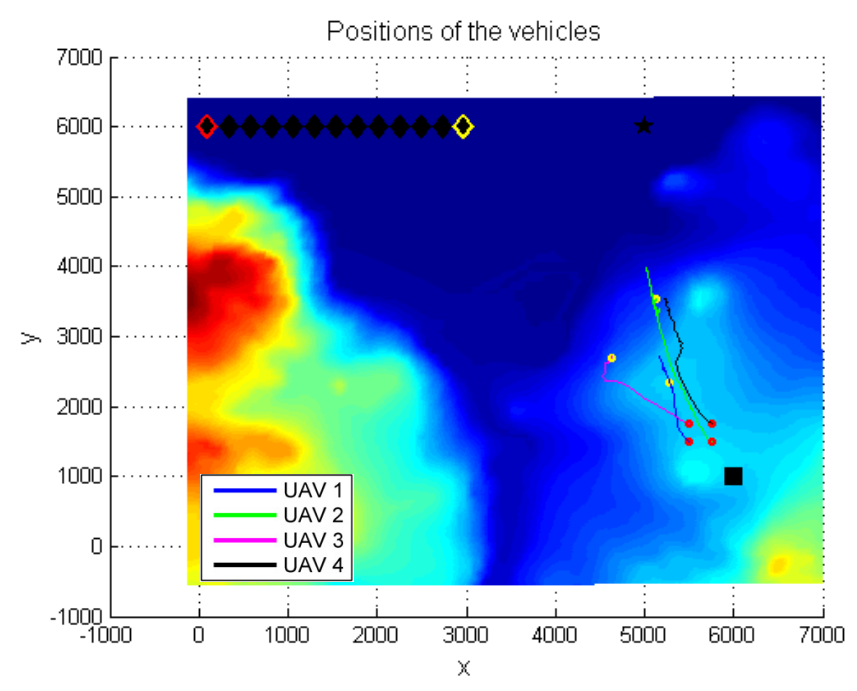

Fig. 5. Trajectories of the four UAVs and the moving target (diamond) - the initial positions are marked with red and the final positions are with yellow, and the positions of the base station (square) and the stationary target (pentagram).

The non-smoothness of the path loss curve in Fig. 16 is due to the fact that the position of the moving target $\mathrm{T} 1$ is updated every 30 [s].

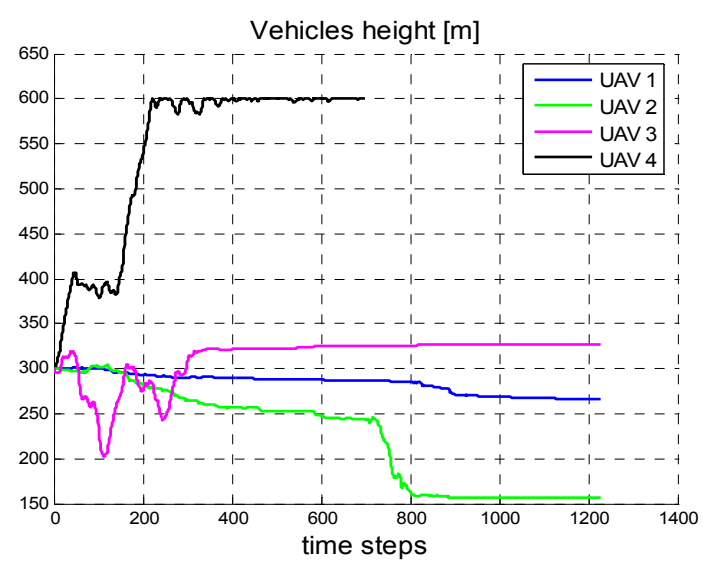

Fig. 6. Heights of the UAVs.

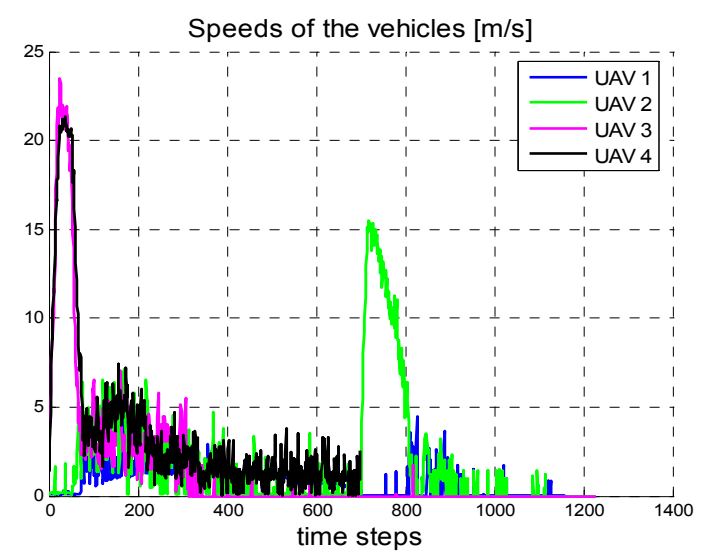

Fig. 7. Speeds of the UAVs.
Path loss between the UAV1 and the base [dB]

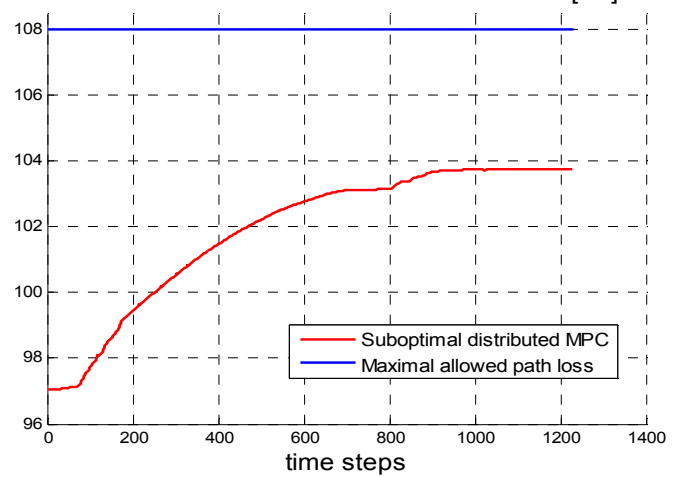

Fig. 8. Radio communication path loss between the UAV1 and the base station.

Path loss between the UAV2 and the base [dB]

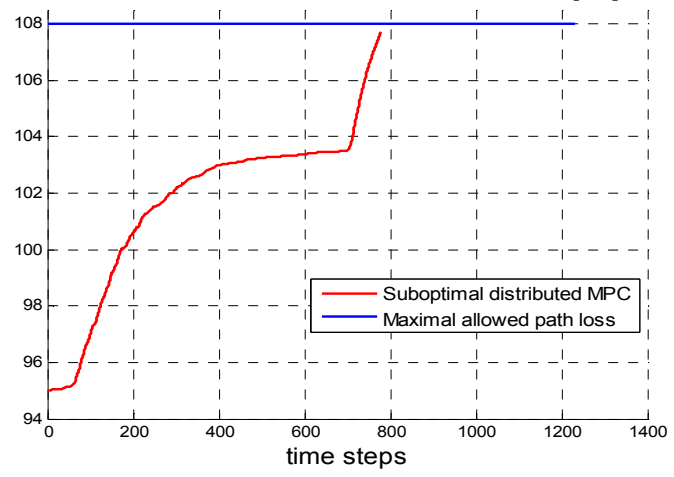

Fig. 9. Radio communication path loss between the UAV2 and the base station.

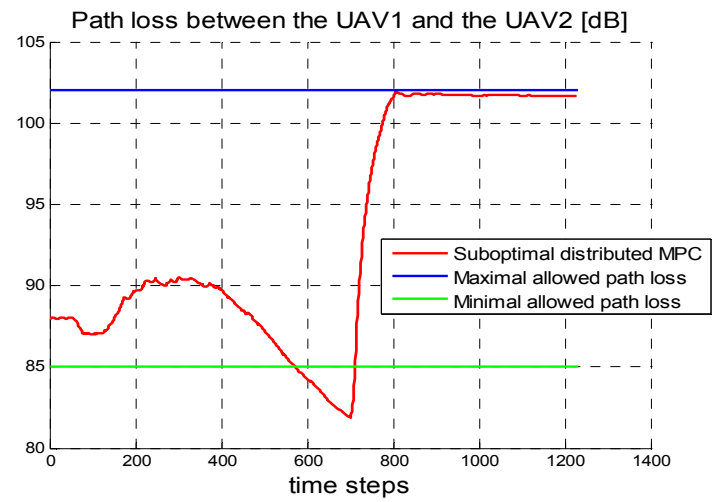

Fig. 10. Radio communication path loss between the UAV1 and the UAV2.

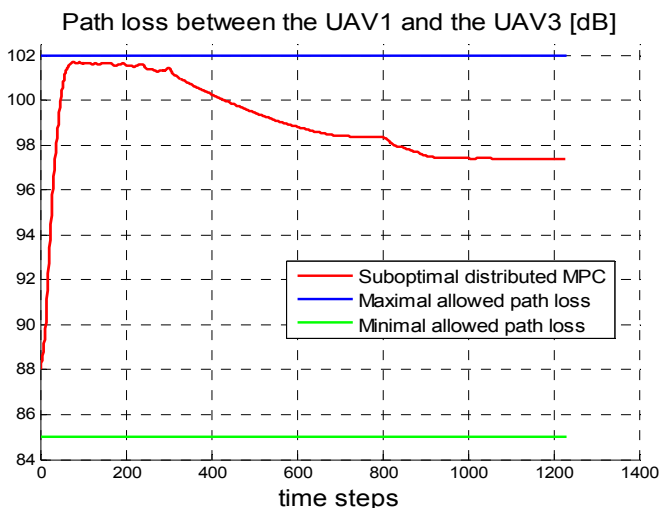

Fig. 11. Radio communication path loss between the UAV1 and the UAV3. 
Path loss between the UAV1 and the UAV4 [dB]

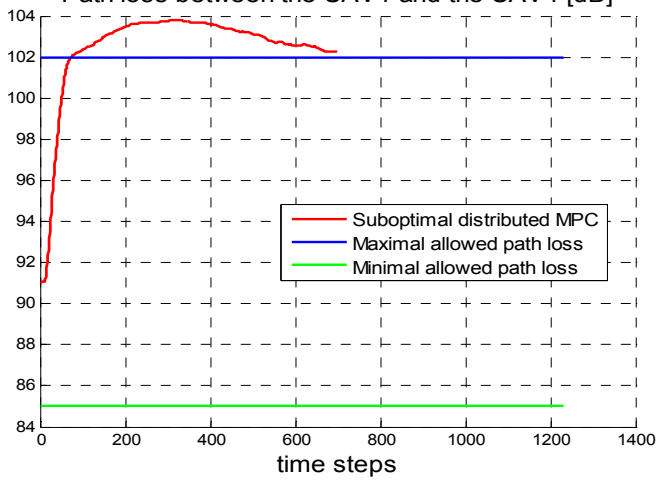

Fig. 12. Radio communication path loss between the UAV1 and the UAV4.

Path loss between the UAV2 and the UAV3 [dB]

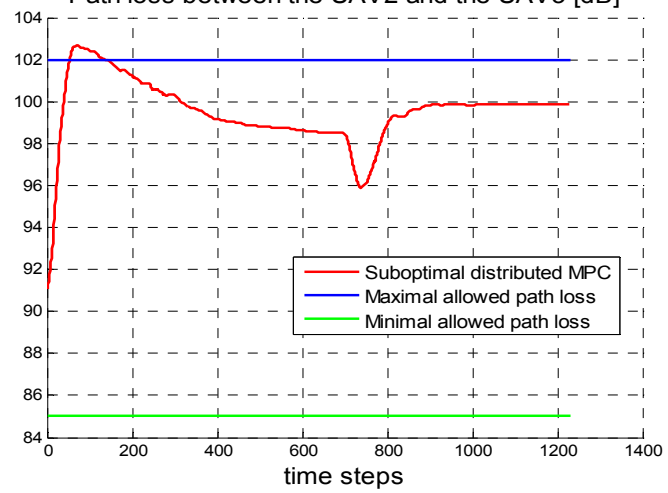

Fig. 13. Radio communication path loss between the UAV2 and the UAV3.

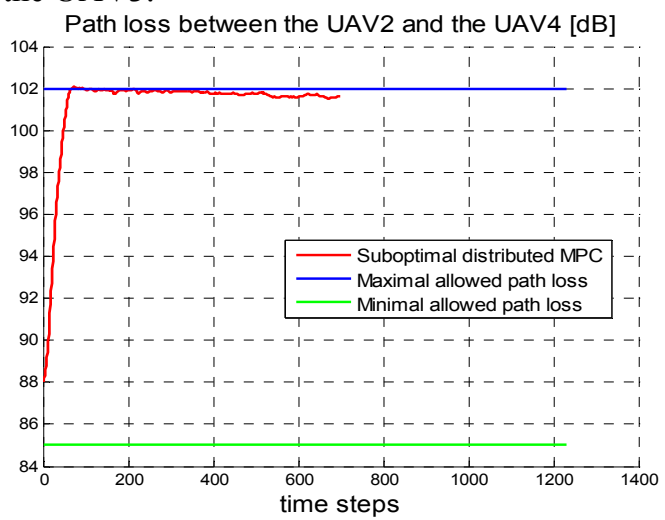

Fig. 14. Radio communication path loss between the UAV2 and the UAV4.

Path loss between the UAV3 and the UAV4 [dB]

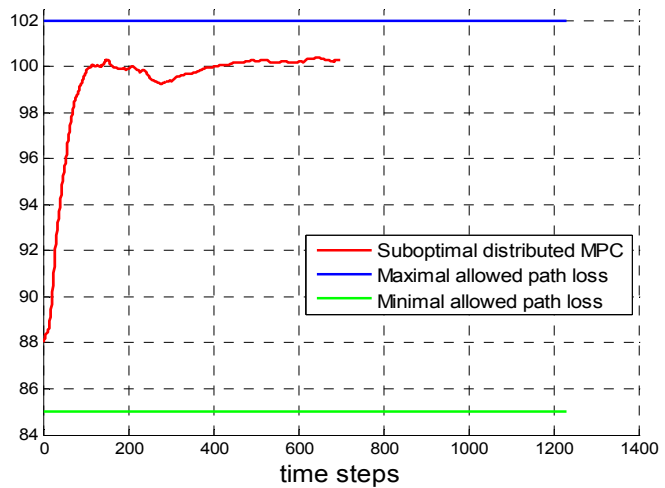

Fig. 15. Radio communication path loss between the UAV3 and the UAV4.

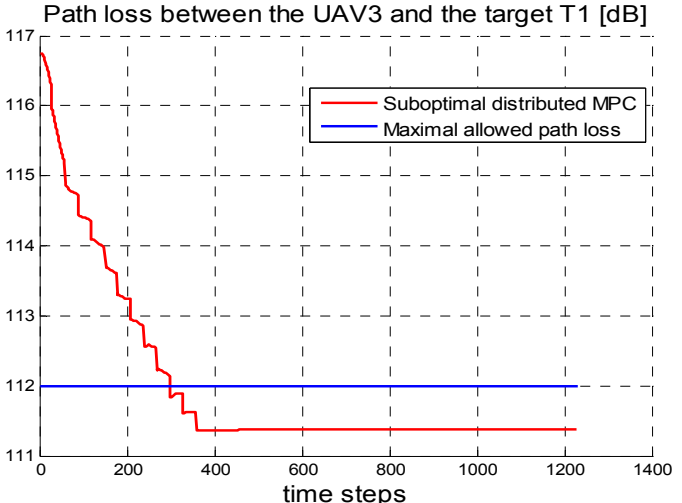

Fig. 16. Radio communication path loss between the UAV3 and the target $\mathrm{T} 1$.

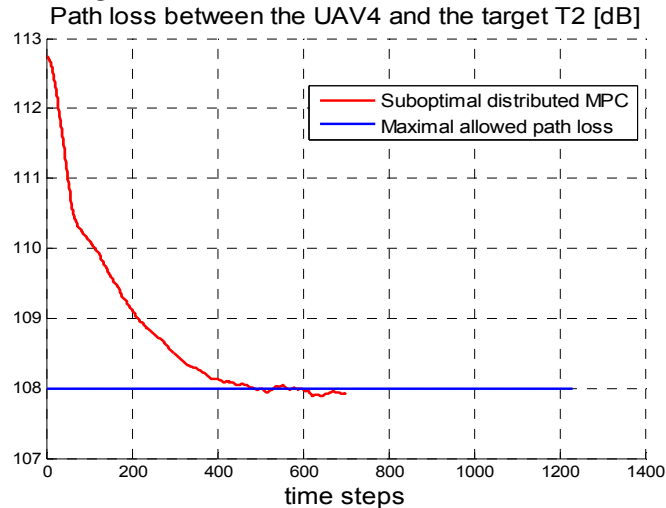

Fig. 17. Radio communication path loss between the UAV4 and the target $\mathrm{T} 2$.

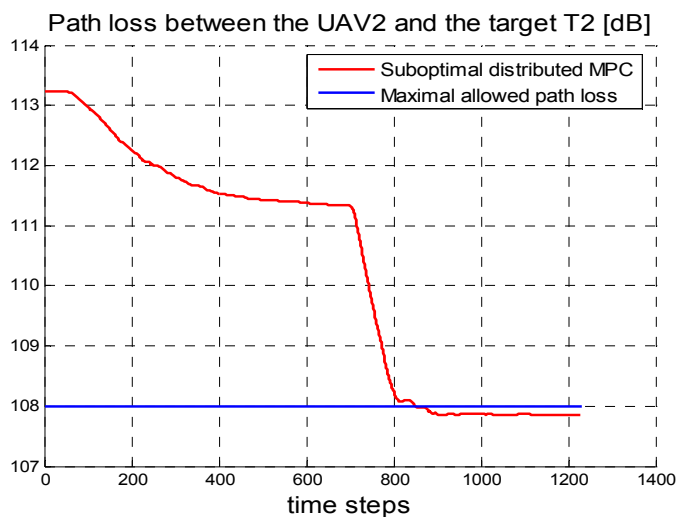

Fig. 18. Radio communication path loss between the UAV2 and the target $\mathrm{T} 2$.

It also should be noted that the use of local linearizations with the suboptimal distributed MPC approach means that in some complex scenarios it may need to be combined with mission planning methods for infeasibility handling based on global information, e.g. (Grøtli and Johansen (2012)).

\section{REFERENCES}

Burdakov, B., Doherty, P., Holmberg, K., Kvarnström, J., and Olsson, P.-M. (2009). Positioning unmanned aerial vehicles as communication relays for surveillance tasks. Robotics Science and Systems, Online Proceedings.

Dixon, C. and Frew, E.W. (2012). Optimizing cascaded chains of unmanned aircraft acting as communication 
relays. IEEE Journal on Selected Areas in Communications, 30, 883-898.

Dunbar, W.B. and Murray, R.M. (2006). Distributed receding horizon control for multi-vehicle formation stabilization. Automatica, 42, 549-558.

Gan, S.K. and Sukkarieh, S. (2011). Multi-UAV target search using explicit decentralized gradient-based negotiation. In Proc. of the IEEE International Conference on Robotics and Automation, Shanghai, China, 2011, pp. 751-756.

Giselsson, P. and Rantzer, A. (2010). Distributed model predictive control with suboptimality and stability guarantees. In Proc. Conference on Decision and Control, Atlanta, GA, pp. 7272-7277.

Grancharova, A., Grøtli, E.I., and Johansen, T.A. (2012a). Distributed MPC-based path planning for UAVs under radio communication path loss constraints. In Proc. of $1 \mathrm{st}$ IFAC Conference on Embedded Systems, Computational Intelligence and Telematics in Control, 2012, Würzburg.

Grancharova, A., Grøtli, E.I., and Johansen, T.A. (2012b). Distributed path planning for a UAV communication chain by dual decomposition. In Proc. of 2-nd IFAC Workshop on Multivehicle Systems, 2012, Espoo, Finland.

Grøtli, E.I. and Johansen, T.A. (2012). Path planning for UAVs under communication constraints using SPLAT! and MILP. Journal of Intelligent and Robotic Systems, 65, 265-282.

Han, Z., Swindlehurst, A., and Liu, K. (2009). Optimization of manet connectivity via smart deployment/movement of unmanned air vehicles. IEEE Transactions on Vehicular Technology, 58, 3533-3546.

Kuwata, Y. and How, J.P. (2011). Cooperative distributed robust trajectory optimization using receding horizon MILP. IEEE Transactions on Control Systems Technology, 19, 423-431.

Le Ny, J., Ribeiro, A., and Pappas, G.J. (2012). Adaptive communication-constrained deployment of unmanned vehicle systems. IEEE Journal on Selected Areas in Communications, 30, 923-934.

Longley, A.G. and Rice, P.L. (1968). Prediction of tropospheric radio transmission loss over irregular terrain: A computer method. U. S. Goverment, Tech. Rep.

Maglicane, J. (2010). SPLAT! An RF signal propagation, loss and terrain analysis tool. http://www.qsl.net/kd2bd/splat.html.

Mayne, D.Q., Rawlings, J.B., Rao, C.V., and Scokaert, P.O.M. (2000). Constrained model predictive control: Stability and optimality. Automatica, 36, 789-814.

McMellen, J. (2010). RF propagation modeling with SPLAT! for Windows. http://blog.gearz.net/2007/09/rfpropagation-modeling-with-splat-for.html.

Michael, N. and Kumar V. (2011). Control of ensembles of aerial robots. Proceedings of the IEEE, 99, 1587-1602.

Moses Sathyaraj, B., Jain, L.C., Finn, A., and Drake, S. (2008). Multiple UAVs path planning algorithms: a comparative study. Fuzzy Optimization and Decision Making, 7, 257-267.

Shin, J. and Kim, H.J. (2009). Nonlinear model predictive formation flight. IEEE Transactions on Systems, Man, and Cybernetics-Part A: Systems and Humans, 39, 11161125 . 